\title{
Mate Searching in Caenorhabditis elegans: A Genetic Model for Sex Drive in a Simple Invertebrate
}

\author{
Jonathan Lipton, Gunnar Kleemann, Rajarshi Ghosh, Robyn Lints, and Scott W. Emmons \\ Department of Molecular Genetics, Albert Einstein College of Medicine, Bronx, New York 10461
}

\begin{abstract}
Much of animal behavior is regulated to accomplish goals necessary for survival and reproduction. Little is known about the underlying motivational or drive states that are postulated to mediate such goal-directed behaviors. Here, we describe a mate-searching behavior of the Caenorhabditis elegans male that resembles the motivated behaviors of vertebrates. Adult $C$. elegans males, if isolated from mating partners, will leave the area of a food source and wander about their environment in an apparent search for a mate. When mating partners are present on the food source, males do not wander but remain with them. This behavior is sexually dimorphic for C. elegans and two additional male/hermaphrodite species studied; for these species, hermaphrodites leave food significantly slower than males. In contrast, for three male-female species examined, both males and females left food, in two cases with similar frequency, suggesting coordinate evolution of behavioral dimorphism with hermaphroditism. We use a quantitative behavioral assay to show that $C$. elegans male mate searching is regulated by signals from hermaphrodites and by physiological signals indicating nutritional and reproductive status. We identify genes in the serotonin, insulin, and sex determination pathways that affect the rate of mate searching. These genes may contribute to physiological and reproductive regulatory mechanisms. Our results establish C. elegans as a model genetic animal with a simple nervous system in which neural pathways leading to a motivated behavior may be genetically dissected.
\end{abstract}

Key words: sexual behavior; motivation; sex drive; serotonin; insulin; behavioral mutant

\section{Introduction}

Drives govern the expression of complex animal behaviors, ensuring that in their activities animals are appropriately goaloriented and purposeful. Animals seek to gain nutrition; they avoid danger and search for a favorable physical environment. They try to find sexual partners and mate with them, and they express behaviors that promote success of their offspring. They express complex social behaviors and seasonal behaviors, such as migrations. Expression of complex, goal-oriented behavioral patterns such as these requires the harmonious functioning of many sensory, neural, and motor systems. In vertebrates, motivational states or drives are invoked to explain the expression and choice among alternative, goal-directed behaviors, but given the complexity of most animal nervous systems, little is known about the detailed nature of such drive states (Kupfermann et al., 2000).

The necessity for the coherent organization of behavior applies to tiny invertebrates no less than to larger species. Although

Received May 6, 2004; revised July 12, 2004; accepted July 14, 2004.

This work was supported by National Institutes of Health (NIH) Grant R01 GM066897 to S.W.E. S.W.E is the Siegfried Ullmann Professor of Molecular Genetics. Some strains used in this study were received from the Caenorhabditis Genetics Center, which was sponsored by a grant from the NIH. J.L. was supported by NIH Medical Scientist Training Program Grant T32 GM07288, and G.K. was supported by NIH Training Program in Cellular and Molecular Biology and Genetics Grant 5T32 GM07491. R.L. was the recipient of a National Alliance for Research on Schizophrenia and Depression Young Investigator Award. We thank H. Eckholdt, N. Hall, and R. Azevedo for assistance with statistical analysis. We are grateful to D. H. Hall and members of the laboratory for helpful discussions and comments on this manuscript and to M. Land for assistance with photographing leaving plates.

Correspondence should be addressed to Dr. Scott W. Emmons at the above address. E-mail: emmons@ aecom.yu.edu.

D0I:10.1523/JNEUROSCI.1746-04.2004

Copyright $\odot 2004$ Society for Neuroscience $\quad$ 0270-6474/04/247427-08\$15.00/0 the nervous system of the nematode worm Caenorhabditis elegans comprises only a few hundred neurons (White et al., 1986), C. elegans can express a number of alternative complex behavioral patterns that are regulated to achieve definable goals. Locomotion, for example, which consists of alternating periods of forward and backward swimming segmented by stops and turns (Croll, 1975; Zheng et al., 1999), is regulated by sensory input to avoid obstacles (Chalfie et al., 1985; Kaplan and Horvitz, 1993), to take the individual toward or away from attractive or noxious substances (Pierce-Shimomura et al., 1999; de Bono et al., 2002), to keep a hungry animal on a food source (Sawin et al., 2000), to keep an animal at the temperature where it last found food (Hedgecock and Russell, 1975), or to keep a male near a mating partner (Simon and Sternberg, 2002). Two reproductive behaviors, egg laying by the hermaphrodite (Hardaker et al., 2001) and copulation by the male (Loer and Kenyon, 1993; Liu and Sternberg, 1995), are coordinated with locomotion. Copulation is itself a complex male behavioral mode with the obvious goal of achieving fertilization (Loer and Kenyon, 1993; Liu and Sternberg, 1995). The possibility of performing a genetic analysis in $C$. elegans and similar small invertebrates opens a way to identify genes necessary for the coordination and expression of overall behavioral patterns such as these. These genes will define the mechanisms responsible for switching between different behavioral states and for making the best choice among alternatives in view of environmental and physiological conditions.

Before a male can copulate, he must locate a mating partner. We show here that under appropriate conditions, the C. elegans male expresses a behavioral pattern that appears to be aimed at accomplishing this goal. If an adult male is isolated on a restricted 
food source without hermaphrodites, he will leave the food source and wander about his environment. When a mating partner on a food source is located, further expression of wandering is blocked. We have studied this behavior as a model to identify genes required for expression of a sex-specific behavior governed by a drive. By measuring the rate at which males leave a food source under various conditions, we have defined environmental, physiological, and genetic factors that govern expression of mate-searching behavior. These experiments establish C. elegans as a model genetic system in which to study the nature and regulation of a drive state.

\section{Materials and Methods}

Strains and culture conditions. Nematodes were propagated following published proce-
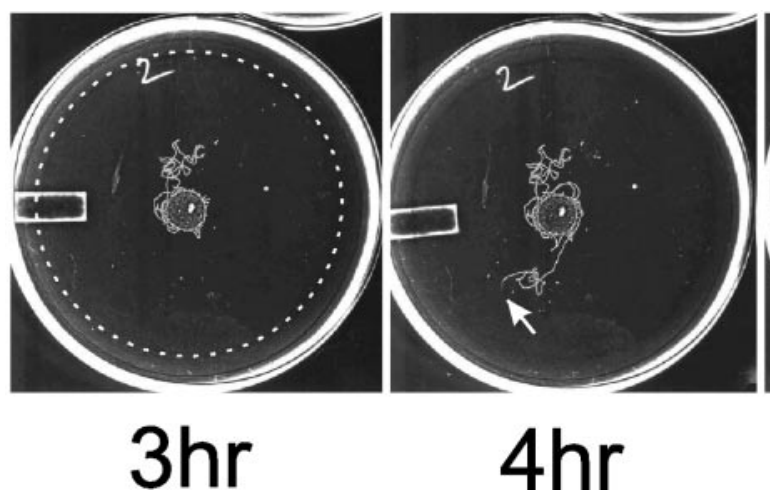

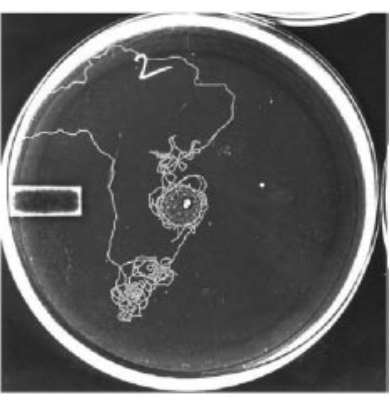

$5 \mathrm{hr}$

Figure 1. Wild-type males leave food. When C. elegans males are isolated on a food source away from mating partners, they eventually leave the food source and wander about their environment. These photographs show the tracks a male left on the agar surface of a leaving assay plate after being placed at time 0 in the center of a $0.9-\mathrm{cm}$-diameter bacterial lawn in the middle of a 9 $\mathrm{cm}$-diameter agar plate. The position of the male on the $4 \mathrm{hr}$ plate is indicated by the white arrow. When the male's track reaches a distance $3 \mathrm{~cm}$ from the edge of the food source (dashed white circle), the male is scored as a leaver ( $5 \mathrm{hr}$ plate). The white rectangle is a raised area of plastic on the bottom of the Petri plate.

dures (Brenner, 1974; Sulston and Hodgkin,

1988). The him-5(e1490) V mutation was introduced into strain backgrounds, where appropriate, to generate cultures with spontaneous males. "Wild type" refers to CB4088 him-5(e1490) animals. The additional $C$. elegans strains used were as follows: CB3844, fem3(e2006)IV; EM653, tph-1(mg280)II; him-5(e1490V); EM670, fem2(b245)III; him-5(e1490)V; EM678, fem-1(hc17)him-8(e1489)IV; EM708, glp-1(bn18)III; him-5(e1490)V; EM709, fog-1(q253)I;him5(e1490)V; EM734, spe-26(hc138)IV; him-5(e1490)V; EM814, daf2(e1370)III; him-5(e1490)V; EM871, daf-16(mgDf50)I; him-5(e1490)V; EM897, daf-16(mgDf50)I;daf-2(e1370)III;him-5(e1490)V; JK577, fog2(q71)V; JK659, mog-3(q74)III; JK816, fem-3(q20)IV.

The strains of other nematode species used were Caenorhabditis briggsae (AF16), Caenorhabditis japonica (SB339), Caenorhabditis remanei (EM464), Caenorhabditis sp. (DF5070), and Pristionchus pacificus (PS312).

Leaving assays. Leaving assays were performed on plastic Petri plates (9-cm-diameter) prepared with $10 \mathrm{ml}$ of agar medium [17 gm of agar (Difco, Detroit, MI), 2.7 gm of Bactopeptone (Difco), $0.55 \mathrm{gm}$ of Tris base (Sigma, St. Louis, MO), 0.27 gm of Tris $\mathrm{HCl}$ (Sigma), $2.0 \mathrm{gm}$ of $\mathrm{NaCl}$ (Fisher Scientific, Pittsburgh, PA), and $1 \mathrm{ml}$ of ethanol containing 5 $\mathrm{mg} / \mathrm{ml}$ cholesterol (Sigma), per liter $\mathrm{H}_{2} \mathrm{O}$ ] and were allowed to dry overnight on a laboratory bench. The smaller amount of agar per plate $(10 \mathrm{ml})$ compared with the standard amount for genetics $(23 \mathrm{ml})$ was used because it was found to increase the ease of scoring worm tracks (see Fig. 1), while not affecting the rate of leaving. Each plate was inoculated in the center with $18 \mu \mathrm{l}$ of Escherichia coli strain OP50 grown to $\mathrm{OD}_{600}=1.0$, establishing after incubation for an additional 12-16 hr at room temperature, a small circular lawn of $\sim 9 \mathrm{~mm}$ diameter. The dryness of the agar surface and degree of bacterial growth were the most critical variables for reproducible results. In a typical assay, L4 larval worms were selected from worm cultures and held on same-sex seeded plates for $\sim 12 \mathrm{hr}$ to mature. Approximately 20 animals were selected, placed individually on assay plates, and scored for leaving (passage of their track beyond a 3.5 $\mathrm{cm}$ radius circle) at intervals. Assays were performed at $20^{\circ} \mathrm{C}$, unless noted otherwise. Tracks were often most easily seen by direct observation of the plate tilted at angles to observe the reflections of laboratory lights on the agar surface. For photography, plates were prepared with $10 \mathrm{ml}$ of nematode growth medium agar (Sulston and Hodgkin, 1988) and photographed following the method of Mori and Ohshima (1995).

Calculation ofleaving rates. The probability of leaving per hour, $P_{\mathrm{L}}$, was estimated in one of two ways: (1) as the slope of the plot of log fraction of nonleavers versus time (see Fig. 2) using a mixed effects repeatedmeasures ANOVA with an autoregressive covariance structure (SAS software, version 6.12; SAS Institute, Inc., Cary, NC); or (2) as the hazard obtained by fitting an exponential parametric survival model to the censored data using maximum likelihood (software R; http://www.
R-project.org). Each condition was contrasted against the appropriate control condition (pooled across replicates).

Food deprivation. Adult male worms were picked from plates seeded with bacteria and washed three times with M9 solution (Brenner, 1974). They were then placed on fresh $9 \mathrm{~cm}$ plates that were either seeded with bacteria or contained agar alone. Plates were placed at $20^{\circ} \mathrm{C}$ for varying lengths of time. Fed or food-deprived individuals were transferred to standard leaving assay plates, and their leaving times were scored.

Gonad ablation. Ablation by laser microsurgery was performed on L1 animals as described (Bargmann and Avery, 1995). Germ-line ablation was accomplished by ablating the germ-line precursor cells Z2 and Z3. Whole gonad ablation was accomplished by ablating these plus the somatic precursors Z1 and Z4. Success of ablations was determined by examining adult animals with Nomarski optics to confirm the absence of the ablated tissues.

\section{Results}

\section{A quantitative assay for mate searching}

In the laboratory, C. elegans is normally reared on agar plates seeded with $E$. coli as a food source. Under these conditions, if an adult male is left alone on a restricted patch of food, he will eventually leave the food source and wander about the plate. We developed a quantitative assay to measure this tendency, a behavior we call "leaving." The assay is based on the time it takes from the start of the assay for a male to traverse a region of the agar plate a certain distance from the food (Fig. 1). Males appear to leave food stochastically, each worm in a population having an equal and invariant probability of leaving in any given time interval (Fig. 2). From the assay, we derive a first-order rate constant, $P_{\mathrm{L}}$ (the probability of leaving per hour), which represents a simple, single, and quantitative means by which to compare genotypes and conditions for the expression of this complex behavior (Fig. 2).

Leaving appears to be a mate-searching behavior for the following reasons. First, leaving behavior is both sex and stage specific. Adult hermaphrodites, which do not need to mate to reproduce, rarely, if ever, wander away from food (Fig. 2, Table 1). Moreover, neither males nor hermaphrodites at the sexually immature L4 stage or earlier express appreciable leaving behavior (Table 1). L4 larval males began leaving after sexual maturation (data not shown). These data suggest that behavioral mechanisms promoting leaving behavior are activated concomitantly with sexual maturation in males. Thus, only animals capable of mating and requiring a mating partner to reproduce leave food. 


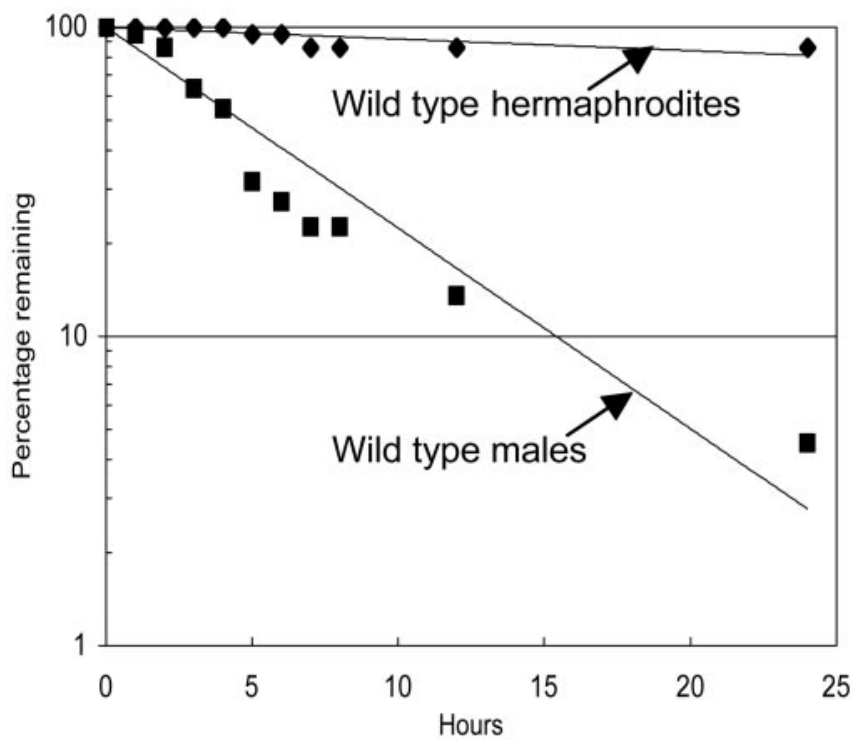

Figure 2. Quantitative measurement of leaving behavior. To determine the probability of leaving food, a number of animals (typically 20) are scored for their time of leaving as shown in Figure 1. A plot is shown of representative data for two assays, one with wild-type C. elegans males and one with wild-type hermaphrodites. The log fraction of nonleavers versus time is plotted, and the data are fitted to the first-order equation $N(t) / N_{0}=\exp \left(-P_{\mathrm{L}} t\right)$, where $N(t) / N_{0}$ is the fraction of total worms that remain nonleavers at time $t$ and $P_{\mathrm{L}}$, the rate constant, represents the probability of leaving per unit time. A reasonable fit to a straight line indicates that to a first approximation, the probability of leaving per unit time is the same for each individual and is constant over the period of the assay.

Second, when adult hermaphrodites are placed on the leaving assay plates with males, the males do not leave food (Fig. $3 A$, Table 1). Thus, encounter of a hermaphrodite has the property of a goal of an appetitive behavior by the criterion that it causes the behavior to cease (Kupfermann et al., 2000). When males are cultured with other males, leaving probability is reduced but not eliminated (Fig. 3A, Table 1). Thus, in the leaving assay, males are capable of distinguishing males from hermaphrodites.

A food supply is necessary for the presence of hermaphrodites to inhibit male leaving. Males tested with paralyzed hermaphrodites $[u n c-51(e 369)$ or $u n c-54(e 1152)]$ in the absence of food but not in the presence of food rapidly left the region containing the hermaphrodites (Table 1). Thus, males are retained by hermaphrodites only in the presence of food, conditions favorable to the production of progeny. Taken together, these observations are consistent with the interpretation that leaving is an adaptive male sexual behavior that promotes finding a mate on food.

\section{Correlation of leaving rates and sexual system}

To determine whether leaving was always correlated with the need to find a mate, we measured leaving rates of the two sexes in additional nematode species. We chose species with both malefemale sexual systems and species with a self-fertile hermaphrodite like C. elegans. The expectation was that only self-fertile hermaphrodites would remain indefinitely on food. Contrary to this expectation, we found a wide range of $P_{\mathrm{L}}$ values for both sexes, suggesting that the balance between wandering and food attraction has evolved (Table 2). Of two hermaphroditic species we tested, neither showed the extreme sexual dimorphism of leaving behavior exhibited by $C$. elegans. Under standard assay conditions, in the closely related hermaphroditic species C. briggsae, both males and hermaphrodites left food at similar rates, whereas in the hermaphroditic species $P$. pacificus, both sexes tended to
Table 1. Leaving probabilities $\left(P_{L}\right)$ of $C$. elegans strains

\begin{tabular}{|c|c|c|}
\hline Genotype or condition & Male & Hermaphrodite \\
\hline \multicolumn{3}{|l|}{ Wild type } \\
\hline Adult & $0.17 \pm 0.008(20)$ & $0.002 \pm 0.001^{a *}(15)$ \\
\hline L4 & $<0.001^{b *}(2)$ & $<0.001(2)$ \\
\hline Male with hermaphrodites & $<0.001^{c *}(8)$ & NA \\
\hline Male with males & $0.103 \pm 0.017^{d *}(3)$ & NA \\
\hline $\begin{array}{l}\text { Male with hermaphrodites, } \\
\text { no food }\end{array}$ & $0.6 \pm 0.05^{e *}(3)$ & NA \\
\hline \multicolumn{3}{|l|}{ Gonad ablations } \\
\hline Entire gonad ablated & $0.05 \pm 0.002^{f_{*}}(n=25)$ & $0.06 \pm 0.002^{f_{*}}(n=23)$ \\
\hline Germ line ablated & $0.07 \pm 0.003^{f_{* *}}(n=18)$ & $0.005 \pm 0.004^{f_{* * *}}(n=30)$ \\
\hline Serotonin biosynthesis & & \\
\hline tph-1(mg280) & $0.04 \pm 0.01^{g *}(3)$ & $0.007 \pm 0.002^{g * * *}(3)$ \\
\hline \multicolumn{3}{|l|}{ Insulin pathway } \\
\hline Wild type & $0.05[0.04,0.06](4)$ & \\
\hline daf-2(e1370) & $0.003[0.001,0.007]^{g *}(4)$ & \\
\hline $\begin{array}{l}\text { daf-16(mgDf50) } \\
\text { daf-2(e1370);daf- }\end{array}$ & $0.11[0.09,0.14]^{g_{*}}(3)$ & $0.003 \pm 0.001^{* * *}$ \\
\hline $16(m g D f 50)$ & $0.06[0.05,0.08]^{g * * *}(3)$ & \\
\hline \multicolumn{3}{|l|}{ Germ cell development $^{g}$} \\
\hline$g l p-1(b n 18)$ & $0.04 \pm 0.002^{*}(2)$ & $<0.001^{* * *}(2)$ \\
\hline spe-26(hc138) & $0.05 \pm 0.005^{*}(2)$ & $0.001 \pm 0.001^{* * *}(2)$ \\
\hline fog-1(q253) & $0.001 \pm 0.001^{*}(3)$ & $0.001 \pm 0.001^{* * *}(2)$ \\
\hline fem-1(hc17) & NA & $0.004 \pm 0.004^{* * *}(2)$ \\
\hline fem-2(b245) & NA & $0.002 \pm 0.002^{* * *}(2)$ \\
\hline fem-3(e2006) & NA & $0.004 \pm 0.003^{* * *}(2)$ \\
\hline fem-3(q20gf) & NA & $0.005 \pm 0.005^{* * *}(3)$ \\
\hline $\operatorname{mog}-3(q 74)$ & NA & $0.010 \pm 0.007^{* * *}(2)$ \\
\hline
\end{tabular}

$P_{\mathrm{L}} \pm$ SE was calculated from the combined data of all independent tests. For the insulin pathway male data, which were analyzed by survival analysis (see Materials and Methods), upper and lower $95 \%$ confidence intervals are given in brackets. The number of independent assays that were combined in the calculation of $P_{\mathrm{L}}$ is given in parentheses. For ablation experiments, the total number of animals examined is given. $p$ values for pairwise contrasts are indicated as follows: ${ }^{*} p<0.001 ;{ }^{* *} p<0.05 ;{ }^{* * *} p>0.05$. For daf-2(e1370);daf-16(mgDf50) versus daf-2(e1370), $p<0.001$; for daf-2(e1370);daf-16(mgDf50) versus daf-16(mgDf50), $p<0.05$. Insulin pathway assays were performed at $25^{\circ} \mathrm{C}$. Wild-type males appear to have a significantly lower rate of leaving at this temperature than at lower temperatures, and this slow leaving apparently requires daf- 16 function. A possible explanation is that the worms are experiencing some stress response at the higher temperature. NA, Not applicable.

${ }^{a-9}$ The data compared were as follows: ${ }^{a}$ wild-type hermaphrodite versus wild-type male; ${ }^{b} \mathrm{~L} 4$ male versus adult male; ${ }^{\text {}}$ male with hermaphrodites versus male alone; ${ }^{d}$ male with males versus male alone; ${ }^{e}$ male with hermaphrodites without food versus male with hermaphrodites on food; ${ }^{f}$ ablated versus unablated; ${ }^{g}$ mutant versus wild type.

remain on the food (data not shown). However, by either increasing ( C. briggsae) or decreasing ( $P$. pacificus) the size of the bacterial lawn, we could demonstrate higher rates of leaving by males than by hermaphrodites in both species (Table 2).

In three male-female species tested, females left food, in one instance [C. sp. (DF5070)] at high rates comparable with males. For two of these species, there was no significant difference in the rates of leaving between males and females. These results are consistent with the need for both sexes to find a mate. Strikingly, for one male-female species, C. remanei, when virgin females were mated, their rate of leaving was decreased (Fig. 3B, Table 2). This indicates that leaving behavior is under the control of signals from the reproductive system and is governed by reproductive status, an observation that provides support for the conclusion that leaving is associated with the requirement to mate.

\section{Regulation of male leaving by a hermaphrodite signal}

Motivated behaviors are governed by cues from the environment and by physiological signals from the tissues that indicate their appropriateness under various circumstances. Mate searching is inappropriate when mates are already present, and, accordingly, C. elegans male mate searching is regulated by a signal from hermaphrodites. The leaving assay allowed us to investigate the nature of this signal.

We found that the hermaphrodite signal appears to be either 
on the hermaphrodite body or is a labile secreted pheromone. Single males were placed with hermaphrodites for $15 \mathrm{hr}$, during which time they had the opportunity to mate. The hermaphrodites were then removed, and male leaving kinetics was determined. After hermaphrodite removal, the rate of male leaving returned to that of isolated virgin males without measurable delay ( $<20 \mathrm{~min}$ ) (Fig. $3 A$ and data not shown). Thus, males could quickly detect the absence of hermaphrodites. If males are attracted by a diffusible signal generated by hermaphrodites, this signal does not persist in the environment at effective levels longer than $\sim 20 \mathrm{~min}$. Alternatively, the males might detect the hermaphrodite by contact. This experiment also demonstrated that neither extensive exposure to the hermaphrodite-derived signal nor previous mating opportunity had an effect on males that persisted in the absence of hermaphrodites.

In view of the possibility that males detected hermaphrodites by contact, we examined whether males on food with hermaphrodites were in continuous contact with them. Spot checks at 1-2 min intervals $(n=600)$ of single males on assay plates $(n=20)$ with five wild-type hermaphrodites revealed that a male was in contact with a hermaphrodite $29 \%$ of the time (4\% mating with spicules inserted into the hermaphrodite vulva, $19 \%$ in contact searching for the vulva, $6 \%$ touching any part of the hermaphrodite body in some other way), whereas for $71 \%$ of the time, a male was not in contact with any part of the hermaphrodite and was moving separately about the lawn. Thus, it is unlikely that male leaving behavior is inhibited by constant contact with hermaphrodites. If males detect hermaphrodites by contact, the effects of such contact must persist during intervals of no contact (measured to have a mean of $6.7 \pm 0.5$ min; range, 3.4-14.2 $\mathrm{min}$ ).

\section{Nutritional status regulates leaving}

The leaving assay sets up a competition between food attraction and exploratory behavior. Therefore, a physiological factor that might be expected to affect the leaving rate is nutritional status. Remaining on food might be favored under conditions of starvation, and we found that this was the case. Males were deprived of food for increasing times, then were placed on leaving assay plates, and their rate of leaving was assayed. After food deprivation, males had a reduced initial rate of leaving (Fig. 3C). After a few hours of feeding on the assay plate, they began to leave. The amount of time required for recovery increased with the duration of previous food deprivation. This demonstrates that leaving is a facultative behavior regulated by conditions specific to individual animals. Its inhibition by food deprivation suggests that the neural mechanism governing its
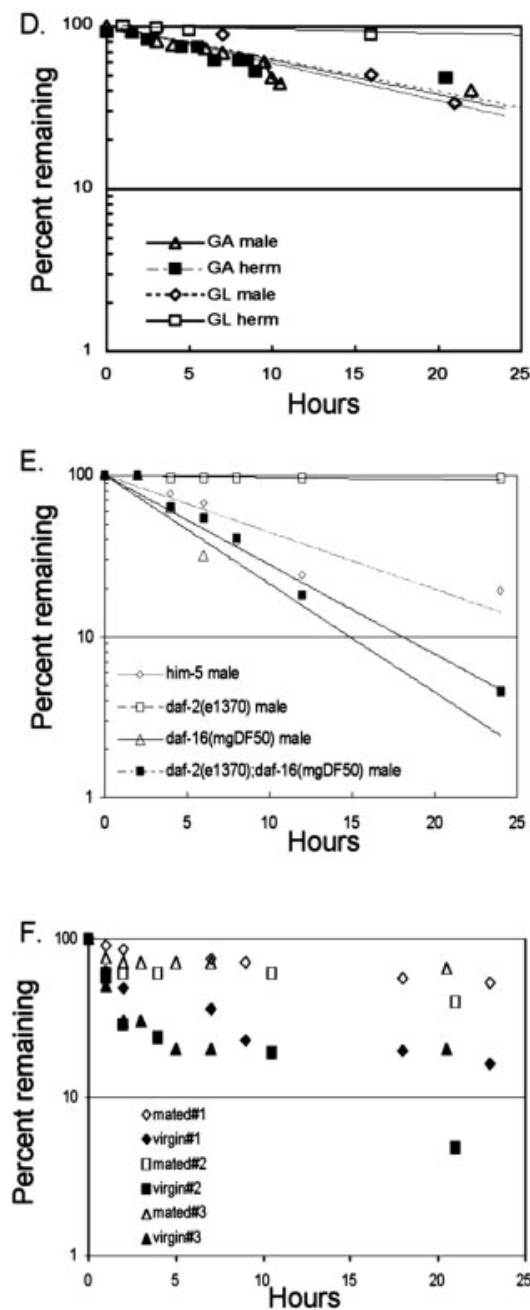

Figure 3. Representative leaving assays under various conditions. $A$, Male leaving is blocked by hermaphrodites and slowed by other males. Single wild-type males were tested in the presence of five wild-type hermaphrodites or paralyzed [unc-51(e364)] the plates. Leaving probability returned to that of males alone without measurable delay, indicating the hermaphrodite signal is on the hermaphrodite body or is labile in the environment. $B$, Leaving behavior in a male-female species, $C$. remanei. Virgin plates without food for the times shown, then tested for leaving behavior. $D$, Effect of the reproductive system on leaving behavior. ther the entire gonad was ablated by laser microsurgery (GA) or the germ line only was ablated (GL). E, Effect of signaling 列 inhibits leaving. A null allele in the downstream transcription factor daf-16(mgDf50) increases leaving and is epistatic to daf-

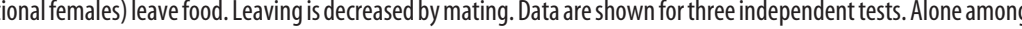
ll genotypes and conditions we tested, leaving data for fog- 2 animals did not yield a straight line for unknown reasons. The $q 71$ allele gave the strongest effects, but we also tested $q 70, q 123,0 z 40$, and oz170 with similar results (data not shown).

expression is responsive to physiological signals reflecting nutritional status.

\section{The reproductive system regulates leaving}

To determine whether another relevant physiological signal came from the gonad, as appeared to be the case in C. remanei, we determined the leaving rates of adult $C$. elegans males and hermaphrodites after gonad ablation. We found that whole-gonad ablation (removal of both germ cells and somatic gonadal tissue) decreased the rate of leaving of males but increased leaving by hermaphrodites (Fig. 3D, Table 1). Rates of leaving by gonadablated males and hermaphrodites were, in fact, indistinguishable, indicating that sexual dimorphism in this behavior requires 
Table 2. Leaving probabilities $\left(P_{L}\right)$ of non-C. elegans species

\begin{tabular}{|c|c|c|}
\hline Species & Male & Hermaphrodite or female \\
\hline \multicolumn{3}{|c|}{ Male/hermaphrodite } \\
\hline C.briggsae & $0.05[0.04,0.07](2)$ & $0.02[0.01,0.03]^{*}(2)$ \\
\hline P. pacificus & $0.05[0.04,0.08](2)$ & $0.006[0.002,0.01]^{*}(2)$ \\
\hline \multicolumn{3}{|l|}{ Male/female } \\
\hline C. japonica & $0.04[0.02,0.06](1)$ & $\begin{array}{l}\text { Virgin: } 0.02[0.01,0.04]^{* * *}(1) \\
\text { Mated: } 0.01[0.006,0.04]^{* * *}(1)\end{array}$ \\
\hline C. remanei & $0.21 \pm 0.07(3)$ & $\begin{array}{l}\text { Virgin: } 0.07 \pm 0.008^{* *}(3) \\
\text { Mated: } 0.03 \pm 0.002^{* *}(3)\end{array}$ \\
\hline C. sp. (DF5070) & $0.27[0.18,0.41](1)$ & $\begin{array}{l}\text { Virgin: } 0.44[0.28,0.68]^{* * *}(1) \\
\text { Mated: } 0.30[0.20,0.45]^{* * *}(1)\end{array}$ \\
\hline
\end{tabular}

For an explanation of values and symbols, see the footnote to Table 1 . The diameter of the food patch was $0.2 \mathrm{~cm}$ fo P. pacificus; $1.0 \mathrm{~cm}$ for C. remanei, C. japonica, and C. sp. (DF5070); and $2.0 \mathrm{~cm}$ for C. briggsae. Pairwise contrasts were hermaphrodite versus male, virgin female versus male, and mated female versus virgin female.

a signal from the reproductive system. It is possible to selectively remove the germ line from the somatic gonad by ablating the two larval germ cell precursors. In males, removal of the germ line decreased the leaving rate similar to whole-gonad ablation, indicating the germ line was critical for the gonad signal (Fig. 3D, Table 1). In hermaphrodites, however, removal of the germ line had no affect on leaving behavior, suggesting that the signal blocking hermaphrodite leaving came from the somatic tissue (Fig. 3D, Table 1). The function of the germ cells in the absence of gonad somatic tissue cannot be tested because germ cell development requires presence of the somatic tissues.

\section{Leaving is regulated by serotonin and by an insulin-like signaling pathway}

Hormones coordinate development, metabolism, reproduction, and behavior. In C. elegans, serotonin and insulin are two signaling pathways known to be involved in the response to food and nutritional status. We found that both pathways affected leaving behavior.

Serotonin is thought to act as a neurohormone after being secreted in response to food into the region of the nerve ring by the neurosecretory NSM cell located in the pharynx (Albertson and Thomson, 1976; Horvitz et al., 1982; Sawin et al., 2000). We found that serotonin-deficient $t p h-1(m g 280)$ males had a significantly decreased rate of leaving (Table 1 ). Serotonin levels might promote leaving as part of a spectrum of responses to nutritional status. Alternatively, or in addition, serotonin might have a more direct involvement in the coordination of locomotion. Eight male-specific CP motor neurons in the ventral cord, thought to innervate the diagonal muscles and required for male copulation, are also serotonergic (Loer and Kenyon, 1993). These neurons represent a possible alternative source of serotonin affecting leaving behavior.

Insulin pathway signaling in C. elegans mediates the choice between rapid development and delayed development (dauer development) and also affects the rate of aging. Pathway activity is influenced by a pheromone, food signals, and signals from the gonad (Kimura et al., 1997; Hsin and Kenyon, 1999). In view of the regulation of male leaving by both food and gonad signaling, we examined whether insulin signaling might be involved. We found that the male leaving rate was dramatically reduced in a mutant for the daf-2 insulin receptor (Fig. 3E, Table 1). daf-2 signaling both to promote nondauer development and to shorten the life span antagonizes the function of the DAF-16 forkhead transcription factor. To determine whether this was the case for behavior as well, we examined the leaving rate of a daf-2; daf-16 double mutant. We found that the daf-16 mutation suppressed the effect of the daf-2 mutation and the animals left at wild-type or even greater rates (Fig. 3E, Table 1). Thus, in a daf-2 loss-offunction background, DAF-16 acts to decrease male leaving. Suppression was incomplete, however, suggesting the possibility that daf-2 may promote leaving by antagonizing an additional activity. None of these mutations affected the hermaphrodite characteristic to remain on food (Table 1 and data not shown) or the ability of males to be retained on food by the presence of hermaphrodites (data not shown). Insulin signaling might act to promote development of nervous system circuitry that enables leaving behavior or it might be required in adult animals to reflect current physiological conditions. In these experiments, performed using a temperature-sensitive daf-2 allele, animals were reared at permissive temperature and raised to nonpermissive temperature either as late L4 animals or as adults. In both cases, males were strongly retarded in leaving. This indicates that pathway activity is required in adult animals.

\section{Genes controlling germ cell development are required for wild-type leaving behavior}

Gonad ablation altered the rate of leaving by both males and hermaphrodites, indicating that one or more signals from the gonad regulated leaving behavior. To confirm and extend these observations, we examined mutants that block or alter germ cell development. Consistent with the result of germ cell ablation in males, $g l p-1$ males, which lack normal proliferation of germ cells, had lower leaving rates than wild-type males (Table 1). Similarly, males defective for spe-26, which have normal numbers of germ cells but are blocked at an early step in sperm differentiation, also had slow leaving rates (Table 1). This suggests that the signal requires the presence of mature male gametes. To determine whether the sex of the gametes was important, we examined males mutant for the sex determination pathway gene fog- 1 , in which male germ cells differentiate as oocytes instead of as sperm (Barton and Kimble, 1990). Remarkably, fog-1 mutant males left food at very low rates, slower than males with no germ cells at all (Table 1). Thus, it appeared that oocytes cause generation of a signal that blocked leaving by males.

Consistent with the lack of any effect in hermaphrodites of ablation of the germ line, most mutations that affected germ cell development had no effect on the tendency of hermaphrodites to remain on food. The mutations tested included mutations that blocked germ-line proliferation $(g l p-1)$, prevented development of hermaphrodite sperm ( fog-1, fem-1, fem-2, fem-3, spe-26), or transformed hermaphrodite gametes entirely into sperm [ $m o g-3$, fem-3(gf)] (Table 1). Thus, C. elegans hermaphrodites were not comparable with mated females of a male-female species $(C$. remanei) in which presence of sperm or developing embryos decreased the rate of leaving.

One gene we tested, however, was exceptional. The gene fog-2 is necessary for the C. elegans hermaphrodite to develop sperm (Schedl and Kimble, 1988). fog-2 acts in the sex determination pathway at an early step to repress pathway activity promoting female development, thereby allowing sperm to be made briefly in the hermaphrodite gonad (Schedl and Kimble, 1988; Clifford et al., 2000). fog-2 virgin hermaphrodites, which lack sperm and are functional females, left food at significant rates (Fig. $3 F$ ). After mating, the rate of leaving was decreased (Fig. $3 F$ ). Therefore, it appears that in a fog-2 mutant background, behavior of the C. elegans hermaphrodite was altered from wild type and can be influenced by the presence of sperm, developing embryos, or possibly a component of seminal fluid.

The effect of $f \circ g-2$ on hermaphrodite behavior is independent 
of the development of male gametes because more downstream mutations that blocked sperm development failed to have any effect on hermaphrodite leaving behavior. Thus, in addition to its function in determining the sex of the gametes, fog-2 also has an independent function that affects behavior. In determining germ-line sex, fog- 2 blocks translation of the tra-2 sex determination gene by acting within a protein complex that targets a $3^{\prime}$ untranslated region element within tra-2 mRNA (Clifford et al., 2000). fog-2 may have additional translational targets allowing it to also block generation of a germ-line signal that would otherwise function to promote leaving in female nematodes.

\section{Discussion}

Mate searching in C. elegans: an appetitive behavior governed by a drive

We have described a behavior of the $C$. elegans adult male that appears to be a mate-searching behavior. This spontaneous, apparently goal-directed behavior is regulated by signals from the environment indicating the presence of hermaphrodite mating partners as well as by internal physiological signals reflecting nutritional and reproductive status. We introduced a simple, quantitative assay that makes it possible to analyze the effects of mutations on expression of this behavior. C. elegans male mate searching provides a potential genetic model for understanding the nature of the signals and signaling pathways that influence expression of a complex, goal-directed behavior.

In vertebrates, the state of the nervous system that influences expression of behavior of this type is termed a motivational or drive state (Kupfermann et al., 2000). Experience-dependent modulation of response to food (Sawin et al., 2000) and environmentally regulated choice of locomotory state (Fujiwara et al., 2002) have been suggested previously as examples of motivational phenomena in C. elegans. A behavioral state model involving regulation by serotonin was used by Waggoner et al. (1998) to describe egg-laying behavior. We suggest that the male can exist in one of two behavioral states, one that promotes leaving and one that prevents it, and that regulation of male mate searching represents an additional example of motivation in C. elegans.

In mate searching, a C. elegans male wanders about his environment. If in wandering he encounters a hermaphrodite on food, he stops wandering and stays indefinitely on this food source for as long as the hermaphrodite is present. It is this phenomenon that allows us to assign encounter of a hermaphrodite on food as the goal and to consider this an appetitive behavior (Kupfermann et al., 2000). The probable sex-specific hermaphrodite signal that the male detects is unknown; it could be a secreted substance (Simon and Sternberg, 2002) or a chemical moiety on the hermaphrodite cuticle that the male detects by touching her. Most likely, it is a combination of multiple signals.

\section{The behavioral basis of male mate searching}

Isolated males leave food in a manner consistent with a stochastic process with a constant probability. This probability might be the probability of switching between two behavioral states, one that favors food attraction and one that results in rapid leaving (e.g., one that suppresses turning and results in long excursions in a straight line or one that momentarily blocks food sensation). Alternatively, a constant probability of leaving might be the random outcome of a single behavioral state the male enters when hermaphrodites are not present.

Patterns of locomotion on and off food have been analyzed for the hermaphrodite (Croll, 1975; Brockie et al., 2001; Hardaker et al., 2001; Fujiwara et al., 2002). On food, hermaphrodite locomotion alternates between two states, one characterized by relatively uninterrupted forward runs (roaming) and one characterized by more frequent turning that keeps the worm within a more restricted area (dwelling) (Fujiwara et al., 2002). Switches between roaming and dwelling states are influenced by sensory input (Fujiwara et al., 2002).

The locomotory behavior of the male has not been studied in similar detail. We do not believe male leaving is simply a consequence of a greater rate of male locomotion because we found no correlation between locomotory rate and leaving rate in a number of mutants we examined (data not shown). The pattern of tracks the male leaves on an agar surface suggests that male locomotion off food might be characterized by roaming and dwelling states similar to the two states of hermaphrodite locomotion on food (Fig. 1). Absence of hermaphrodites might cause males to favor the roaming state. In chemical gradients off food, the pattern of runs and turns made by hermaphrodites is influenced by changing chemical concentration (PierceShimomura et al., 1999). Because males are also expected to be attracted toward food, leaving might be promoted via regulation of male responsiveness to attractive food signals. Simon and Sternberg (2002) have demonstrated that when in the vicinity of hermaphrodites, males have an increased rate of reversals. This increase might be sufficient to account for their failure to leave the food.

\section{Regulation of behavior by nutritional status}

Absence of food or starvation affect several C. elegans behaviors, including the rate of locomotion (Croll, 1975; Croll and Smith, 1978; Sawin et al., 2000), pharyngeal pumping (Avery and Horvitz, 1990), defecation (Liu and Thomas, 1994), egg laying (Chalfie and White, 1988), thermotaxis (Hedgecock and Russell, 1975; Mori, 1999), and olfaction (Colbert and Bargmann, 1997). Serotonin is thought to be one important food-associated signal mediating these effects (Horvitz et al., 1982; Trent et al., 1983; Avery and Horvitz, 1990; Sawin et al., 2000; Sze et al., 2000).

In humans and other animals, serotonin has powerful effects on modulation of behavior and mood (Hull et al., 1999, 2002). In C. elegans, it might be involved in regulating transitions between behavioral states. Such a role has been demonstrated for behavioral states involved in egg laying (Waggoner et al., 1998) and in coordination of egg laying and locomotion (Hardaker et al., 2001). Therefore, the effect of serotonin on male leaving might be attributable to a role of this neurotransmitter in modulating transitions to the mate-searching behavioral state. However, serotonin also affects locomotion itself (Horvitz et al., 1982; Sze et al., 2000; Hardaker et al., 2001), and serotonin-deficient animals leave altered track patterns on agar (our unpublished observations). By affecting the pattern of runs and turns, serotonin might have a more direct effect on the rate at which males can leave.

A second pathway that coordinates multiple aspects of $C$. elegans growth, development, and metabolism is the daf-2/ daf-16 insulin pathway. Signaling through this pathway is necessary for normal reproductive development in the presence of food, whereas loss of such signaling results in the developmental and metabolic shift to the diapause dauer state that follows crowding and low food levels (Riddle and Albert, 1997). Signaling through this pathway also mediates the effect of signals derived from the reproductive system that affect life span (Hsin and Kenyon, 1999) as well as the effects of altered sero- 
tonin levels on development and life span (Sze et al., 2000). Here, we show function of this pathway during adulthood also affects behavior, being necessary to promote mate searching in males. Hence, insulin signaling may be involved in the coordination of an appetitive sexual behavior with metabolic and reproductive status.

\section{Regulation of behavior by the reproductive system}

Not surprisingly, for a reproductive behavior, mate searching is regulated by signals from the gonad. Gonad ablation resulted in opposite effects in males and hermaphrodites, decreasing the leaving rate in the former and increasing the leaving rate in the latter. As a result, gonad-ablated males and hermaphrodites left food at similar rates. Hence, sexual dimorphism of this behavior is entirely dependent on differential signaling from the gonad. The mature gonad makes up a large fraction of the mass of the $C$. elegans adult body, and rapid reproduction appears to be a significant life-history characteristic of this species (Hodgkin and Barnes, 1991). Hence, it may be expected that the gonad plays a central role in coordinating and dictating the functions of the somatic tissues (e.g., it is possible that the signal that activates leaving behavior after maturation of an L4 male to an adult is a gonadal signal). Previously, gonad signaling has been demonstrated to effect C. elegans life span (Hsin and Kenyon, 1999) and growth (Patel et al., 2002).

We have demonstrated that in both males and hermaphrodites, germ cells play a role in gonad signaling that affects leaving behavior. Males in which the sex of the germ line was transformed from male to female by mutation of fog- 1 remained on food, whereas hermaphrodites in which development of male germ cells was blocked by mutation in fog- 2 left food. Both fog- 1 and fog-2 are thought to be germ line limited in their expression (Clifford et al., 2000; Jin et al., 2001). Because there was no effect of ablation of the germ cells in wild-type hermaphrodites, our results suggest that the function of $f \circ g-2$ must be to prevent signaling from germ cells that promotes leaving. As an early-acting gene in the sex determination hierarchy, by regulating both mate-searching behavior and the sex of gametes, fog-2 may ensure coordinate expression of sexual and behavioral phenotypes.

\section{References}

Albertson DG, Thomson JN (1976) The pharynx of Caenorhabditis elegans. Philos Trans R Soc Lond B Biol Sci 275:299-325.

Avery L, Horvitz HR (1990) Effects of starvation and neuroactive drugs on feeding in Caenorhabditis elegans. J Exp Zool 253:263-270.

Bargmann CI, Avery L (1995) Laser killing of cells in Caenorhabditis elegans. In: Methods in cell biology, Vol 48, Caenorhabditis elegans: modern biological analysis of an organism (Epstein HF, Shakes DC, eds), pp 225-250. San Diego: Academic.

Barton MK, Kimble J (1990) fog-1, a regulatory gene required for specification of spermatogenesis in the germ line of Caenorhabditis elegans. Genetics 125:29-39.

Brenner S (1974) The genetics of Caenorhabditis elegans. Genetics 77:71-94.

Brockie PJ, Mellem JE, Hills T, Madsen DM, Maricq AV (2001) The C. elegans glutamate receptor subunit NMR-1 is required for slow NMDAactivated currents that regulate reversal frequency during locomotion. Neuron 31:617-630.

Chalfie M, White J (1988) The nervous system. In: The nematode Caenorhabditis elegans (Wood W, ed), pp 337-391. New York: Cold Spring Harbor Laboratory.

Chalfie M, Sulston JE, White JG, Southgate E, Thomson JN, Brenner S
(1985) The neural circuit for touch sensitivity in Caenorhabditis elegans. J Neurosci 5:956-964.

Clifford R, Lee M, Nayak S, Ohmachi M, Giorgini F, Schedl T (2000) FOG-2, a novel F-box containing protein, associates with the GLD-1 RNA binding protein and directs male sex determination in the C. elegans hermaphrodite germline. Development 127:5265-5276.

Colbert HA, Bargmann CI (1997) Environmental signals modulate olfactory acuity, discrimination, and memory in Caenorhabditis elegans. Learn Mem 4:179-191.

Croll NA (1975) Components and patterns in the behavior of the nematode Caenorhabditis elegans. J Zool (Lond) 176:159-176.

Croll NA, Smith JM (1978) Integrated behavior in the feeding phase of Caenorhabditis elegans. J Zool (Lond) 184:507-517.

de Bono M, Tobin DM, Davis MW, Avery L, Bargmann C (2002) Social feeding in Caenorhabditis elegans is induced by neurons that detect aversive stimuli. Nature 419:899-903.

Fujiwara M, Sengupta PL, McIntire SL (2002) Regulation of body size and behavioral state of C. elegans by sensory perception and the EGL-4 cGMPdependent protein kinase. Neuron 36:1091-1102.

Hardaker LA, Singer E, Kerr R, Zhou G, Schafer WR (2001) Serotonin modulates locomotory behavior and coordinates egg-laying and movement in Caenorhabditis elegans. J Neurobiol 49:303-313.

Hedgecock EM, Russell RL (1975) Normal and mutant thermotaxis in the nematode Caenorhabditis elegans. Proc Natl Acad Sci USA 72:4061-4065.

Hodgkin JA, Barnes TM (1991) More is not better: brood size and population growth in a self-fertilizing nematode. Proc R Soc Lond B Biol Sci 246:19-24.

Horvitz HR, Chalfie M, Trent C, Sulston JE, Evans PD (1982) Serotonin and octopamine in the nematode Caenorhabditis elegans. Science 216:1012-1014.

Hsin H, Kenyon C (1999) Signals from the reproductive system regulate the lifespan of C. elegans. Nature 399:362-366.

Hull EM, Lorrain DS, Du J, Matuszewich L, Lumley LA, Putnam SK, Moses J (1999) Hormone-neurotransmitter interactions in the control of sexual behavior. Behav Brain Res 105:105-116.

Hull EM, Meisel RL, Sachs BD (2002) Male sexual behavior. In: Hormones, brain and behavior (Pfaff DW, Arnold AP, Fahrbach SE, Etgen AM, Rubin RT, eds), pp 3-137. San Diego: Academic.

Jin S-W, Kimble J, Ellis RE (2001) Regulation of cell fate in Caenorhabditis elegans by a novel cytoplasmic polyadenylation element binding protein. Dev Biol 229:537-553.

Kaplan J, Horvitz HRH (1993) A dual mechanosensory and chemosensory neuron in Caenorhabditis elegans. Proc Natl Acad Sci USA 90:2227-2231.

Kimura KD, Tissenbaum HA, Liu Y, Ruvkun G (1997) daf-2, an insulin receptor-like gene that regulates longevity and diapause in Caenorhabditis elegans. Science 277:942-946.

Kupfermann I, Kandel ER, Iversen S (2000) Motivational and addictive states. In: Principles of neural science, Ed 4 (Kandel ER, Schwarz JH, Jessell TM, eds), pp 998-1013. New York: Elsevier.

Liu DWC, Thomas JH (1994) Regulation of a periodic motor program in C. elegans. J Neurosci 14:1953-1962.

Liu KS, Sternberg PW (1995) Sensory regulation of male mating behavior in Caenorhabditis elegans. Neuron 14:1-20.

Loer CM, Kenyon CJ (1993) Serotonin-deficient mutants and male mating behavior in the nematode Caenorhabditis elegans. J Neurosci 13:5407-5417.

Mori I (1999) Genetics of chemotaxis and thermotaxis in the nematode Caenorhabditis elegans. Annu Rev Genet 33:399-422.

Mori I, Ohshima Y (1995) Neural regulation of thermotaxis in Caenorhabditis elegans. Nature 376:344-348.

Patel MN, Knight CG, Karageorgi C, Leroi AM (2002) Evolution of germline signals that regulate growth and aging in nematodes. Proc Natl Acad Sci USA 99:769-774.

Pierce-Shimomura JT, Morse TM, Lockery SR (1999) The fundamental role of pirouettes in Caenorhabditis elegans chemotaxis. J Neurosci 19:9557-9569.

Riddle DL, Albert PS (1997) Genetic and environmental regulation of dauer larva development. In: C. elegans II (Riddle DL, Blumenthal T, Meyer BJ, Priess JR, eds), pp 739-768. New York: Cold Spring Harbor Laboratory. 
Sawin ER, Ranganathan R, Horvitz HR (2000) C. elegans locomotory rate is modulated by the environment through a dopaminergic pathway and by experience through a serotonergic pathway. Neuron 26:619-631.

Schedl T, Kimble J (1988) fog-2, a germ-line-specific sex determination gene required for hermaphrodite spermatogenesis in Caenorhabditis elegans. Genetics 119:43-61.

Simon JM, Sternberg PW (2002) Evidence of a mate-finding cue in the hermaphrodite nematode Caenorhabditis elegans. Proc Natl Acad Sci USA 99:1598-1603.

Sulston J, Hodgkin J (1988) Methods. In: The nematode Caenorhabditis elegans (Wood WB, ed), pp 587-606. New York: Cold Spring Harbor Laboratory.

Sze J, Victor M, Loer C, Shi Y, Ruvkun G (2000) Food and metabolic signal- ing defects in a Caenorhabditis elegans serotonin-synthesis mutant. $\mathrm{Na}$ ture 403:560-564.

Trent C, Tsung N, Horvitz HR (1983) Egg-laying defective mutants of the nematode Caenorhabditis elegans. Genetics 104:619-647.

Waggoner LE, Zhou GT, Schafer RW, Schafer WR (1998) Control of alternative behavioral states by serotonin in Caenorhabditis elegans. Neuron 21:203-214.

White JG, Southgate E, Thomson JN, Brenner S (1986) The structure of the nervous system of Caenorhabditis elegans. Philos Trans R Soc Lond B Biol Sci 314:1-340.

Zheng Y, Brockie J, Mellem JE, Madsen DM, Maricq AV (1999) Neuronal control of locomotion in C. elegans is modified by a dominant mutation in the GLR-1 ionotorpic glutamate receptor. Neuron 24: 347-361. 